\title{
CCR2 is localized in microglia and neurons, as well as infiltrating monocytes, in the lumbar spinal cord of ALS mice
}

Hiroyasu Komiya, Hideyuki Takeuchi ${ }^{*}$ D, Yuki Ogawa, Yuki Hatooka, Keita Takahashi, Atsuko Katsumoto, Shun Kubota, Haruko Nakamura, Misako Kunii, Mikiko Tada, Hiroshi Doi and Fumiaki Tanaka*

\begin{abstract}
It remains controversial whether circulating monocytes expressing CCR2 infiltrate the central nervous system (CNS) and contribute to pathogenicity of amyotrophic lateral sclerosis (ALS). A previous report used conventional immunohistochemistry to show that CCR2 is exclusively expressed by astrocytes, but not infiltrating monocytes/ microglia or neurons, in the spinal cords of ALS model mice. In this study, we assessed the cellular distribution of

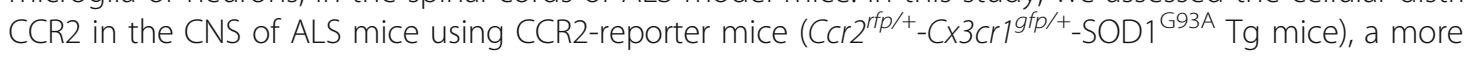
sophisticated method for directly detecting the distribution of CCR2 protein. We found that infiltration of CCR2 ${ }^{+}$ monocytes in the lumbar spinal cord increased over the course of disease progression. Moreover, from the middle stage of disease, CCR2 was partially distributed in microglia and neurons, but not astrocytes, in striking contrast to the previous findings. These novel observations suggested that CCR2 ${ }^{+}$monocyte infiltration leads to CNS environmental deterioration due to toxic conversion of microglia and neurons, creating a vicious cycle of neuroinflammation and leading to acceleration of ALS pathology. Our findings also show that this reporter mouse is a useful and powerful tool for obtaining new insights into the pathomechanisms of ALS.
\end{abstract}

Keywords: Amyotrophic lateral sclerosis, CCR2, Astrocyte, Neuron, Microglia, Monocyte

\section{Main text}

Amyotrophic lateral sclerosis (ALS) is a fatal neurodegenerative disease characterized by the selective loss of motor neurons in the central nervous system (CNS). The nonautonomous neuronal death hypothesis, based on recent studies, states that neuroinflammation by nonneuronal neighboring cells, such as glia and infiltrating cells, is critical for disease progression in ALS [1]. In general, tissue inflammation induces migration of circulating monocytes, especially chemokine CC motif receptor 2 (CCR2) ${ }^{\text {high }}$ monocytes [2]. It remains controversial whether CCR2 ${ }^{\text {high }}$ monocytes infiltrate the CNS and contribute to ALS

\footnotetext{
* Correspondence: htake@yokohama-cu.ac.jp; ftanaka@yokohama-cu.ac.jp Department of Neurology and Stroke Medicine, Yokohama City University Graduate School of Medicine, 3-9 Fukuura Kanazawa-ku, Yokohama 236-0004, Japan
}

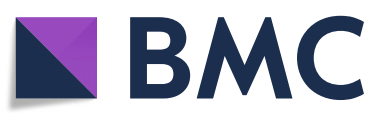

pathogenesis [3, 4]. However, Kawaguchi-Niida et al. showed using conventional immunohistochemistry, that CCR2 is exclusively expressed by astrocytes, but not infiltrating monocytes/microglia or neurons, in the spinal cords of ALS mice [5]. This variability on CCR2 $2^{+}$cellular distribution may partly depend on how CCR2 is detected. For immunostaining, commercially available anti-mouse CCR2 antibodies are usually suitable for flow cytometry, but less so for immunohistochemistry/immunofluorescence, as is usually the case for cell surface antigens. Accordingly, the CCR2-RFP reporter mouse was developed to circumvent this difficulty [2].

In this study, we used this CCR2-reporter mouse to directly detect the distribution of CCR2 protein, with the goal of assessing whether CCR2 ${ }^{+}$monocytes infiltrate the CNS of ALS mice. We used transgenic mice

(c) The Author(s). 2020 Open Access This article is licensed under a Creative Commons Attribution 4.0 International License, which permits use, sharing, adaptation, distribution and reproduction in any medium or format, as long as you give appropriate credit to the original author(s) and the source, provide a link to the Creative Commons licence, and indicate if changes were made. The images or other third party material in this article are included in the article's Creative Commons licence, unless indicated otherwise in a credit line to the material. If material is not included in the article's Creative Commons licence and your intended use is not permitted by statutory regulation or exceeds the permitted use, you will need to obtain permission directly from the copyright holder. To view a copy of this licence, visit http://creativecommons.org/licenses/by/4.0/ The Creative Commons Public Domain Dedication waiver (http://creativecommons.org/publicdomain/zero/1.0/) applies to the data made available in this article, unless otherwise stated in a credit line to the data. 
carrying a high copy number of a transgene encoding a G93A mutant of human superoxide dismutase 1 (SOD1 ${ }^{\text {G93A }} \mathrm{Tg}$ mice; \#004435, Jackson Laboratory) as an ALS model. We obtained $\mathrm{Ccr}_{2} \mathrm{fp} /+_{-} \mathrm{Cx} 3 \mathrm{cr} 1^{g \mathrm{fp} /+_{-}}$ SOD $1^{\text {G93A }} \mathrm{Tg}$ mice by crossing $\mathrm{SOD}^{\mathrm{G} 93 \mathrm{~A}} \mathrm{Tg}$ mice with $C c r 2^{r f p / r f p}$ mice (\#017586, Jackson Laboratory) and $C x 3 c r 1^{g f p / g f p}$ mice (\#005582, Jackson Laboratory), which enables us to easily differentiate $\mathrm{CCR} 2^{+}$infiltrating monocytes from $\mathrm{CX} 3 \mathrm{CR} 1^{+}$tissue-resident macrophages (i.e., microglia in the CNS) [2, 6]. These mice were backcrossed for more than 10 generations after purchase. Histological analysis was carried out using $20-\mu \mathrm{m}$-thick frozen sections of lumbar spinal cords in $\mathrm{Ccr} 2^{r f p /+}$ $\mathrm{C} \times 3 \mathrm{Cr}^{8 f p /+}-\mathrm{SOD} 1^{\mathrm{G} 93 \mathrm{~A}} \mathrm{Tg}$ mice. Sections were permeabilized with $1 \%$ Triton X-100 after blocking with $10 \%$ normal goat serum for $30 \mathrm{~min}$, and then stained with specific primary antibodies for markers of neurons (NeuN; \#MAB377, Chemicon, Temecula, CA, USA), monocytes/microglia (Iba1; \#019-19,741, Wako, Osaka, Japan), and astrocytes (GFAP; \#M0761, Dako, Glostrup, Denmark), followed by Alexa Fluor 488-conjugated secondary antibodies (Molecular Probes, Eugene, OR, USA). Images were analyzed on a deconvolution fluorescence microscope (BZ-9000, Keyence, Osaka, Japan), and quantitative analyses of immunopositive cells in the lumbar spinal cord (6 sections/mouse, $n=5$ ) were carried out using ImageJ as described [7]. Statistical significance was determined by one-way analysis of variance (ANOVA) followed by post-hoc Tukey's test using Prism version 7.0 (GraphPad Software, La Jolla, CA, USA).

$C \mathrm{Cr} 2^{r f p /+}-C_{2} 3 \mathrm{crr}^{g f p /+}{ }_{-} \mathrm{SOD} 1^{\text {G93A }} \mathrm{Tg}$ mice exhibited similar disease progression to SOD $1^{\mathrm{G} 93 \mathrm{~A}} \mathrm{Tg}$ mice (median survival days $=167$ vs. 160 , respectively; Fig. 1), and the abundance of CX3CR $1^{+}$microglia in lumbar spinal cord gradually increased as the disease progressed (Supplemental Figure 1), consistent with the previous reports $[7,8]$. Chronological assessments also revealed that the abundance of $\mathrm{CCR} 2^{+}$cells gradually increased in the gray matter of the lumbar spinal cord as disease progressed (Fig. $1 \mathrm{~b}$ and $\mathrm{c}$ ), whereas CNS infiltration by $\mathrm{CCR} 2^{+}$cells was not detected in $\mathrm{Ccr} 2^{r f p /+}-\mathrm{Cx} 3 \mathrm{cr} 1^{g f p /+}$ mice. Next, we evaluated the cellular localization of CCR2 by immunofluorescence staining for NeuN (neuron marker), Iba1 (monocytes and microglia marker), and GFAP (astrocyte marker). Most of CCR2 $2^{+}$cells were positive for Iba1 (Fig. $1 \mathrm{~d}$ and $\mathrm{h}$ ), but not for CX3CR1, until the middle stage of the disease, suggesting that most CCR2 ${ }^{+}$cells in the CNS were infiltrating monocytes at early disease stages. These findings corresponded with a previous study reporting that $\mathrm{CCR} 2^{+}$monocytes were recruited into the spinal cord of SOD1 ${ }^{\text {G93A }} \mathrm{Tg}$ mice [3]. Surprisingly, from the middle stage of the disease, CCR2 was partially distributed in CX3CR $1^{+}$microglia (Fig. 1e and i) and neurons (Fig. 1f and j), but not in astrocytes (Fig. 1g), in striking contrast to the previous findings by Kawaguchi-Niida et al. [5]. The proportion of each type of CNS-resident cell that was $\mathrm{CCR}^{+}{ }^{+}$increased as disease progressed, whereas the percentage of $\mathrm{CX}_{3} \mathrm{CR}^{+}{ }^{+}$or $\mathrm{Iba}^{+}$cells that was $\mathrm{CCR} 2^{+}$ reached a plateau at the middle stage of the disease (Fig. 1i). No resident CNS cells expressed CCR2 in $\mathrm{Ccr} 2^{r \mathrm{fp} / t_{-}}$ $\mathrm{C} x 3 \mathrm{cr} 1^{g f p /+}$ non-Tg mice (Supplemental Figure 2). These novel observations demonstrated that CCR2 is expressed in resident CNS cells such as microglia and neurons, as well as CNS-infiltrating monocytes, in the advanced stage of ALS.

The cellular distribution and physiological role of CCR2 in the CNS have yet to be elucidated. Previous studies reported that CCR2 is present only in monocytes/macrophages and basophils, whereas Ccr2 mRNA is expressed in most leukocytes, including monocytes/ macrophages, $\mathrm{T}$ cells, $\mathrm{B}$ cells, natural killer cells, basophils, and dendritic cells [2]. However, CCR2 is constitutively expressed in neurons in murine brain, spinal cord, and dorsal root ganglia, and upregulation of CCL2 (the ligand of CCR2)-CCR2 axis in the disease state directly causes neuronal dysfunction through Akt signaling pathway [9-11]. Other studies reported that CCR2 is expressed in both infiltrating monocytes and microglia in a rodent model of traumatic brain injury [12]. Furthermore, another study reported CCR2 ${ }^{+}$monocyte infiltration in the perivascular areas of the primary motor cortex in ALS patients with TDP-43 pathology [13]. These data are discordant with the findings of Kawaguchi-Niida et al., who reported CCR2 expression exclusively in astrocytes [5]. Also in our ALS mice, CCR2-RFP was not detected in astrocytes, but was instead found in CNS-infiltrating monocytes, CX3CR $1^{+}$ microglia, and neurons.

There are two possible origins of $\mathrm{CCR} 2^{+} \mathrm{CX} 3 \mathrm{CR} 1^{+}$ cells in the spinal cord of ALS mice. One possibility is that $\mathrm{CX} 3 \mathrm{CR} 1^{+}$microglia express CCR2. Initially, CCL2 is released from activated microglia and recruits $\mathrm{CCR}^{+}$ monocytes into the spinal cord. Infiltrated CCR2 ${ }^{+}$ monocytes also release CCL2, which accelerates inflammatory cell accumulation and leads to environmental deterioration including neuroinflammation and neuronal dysfunction, further provoking neuronal CCR2 expression. Subsequently, this microenvironmental change might convert $\mathrm{CX}_{3 \mathrm{CR}} 1^{+}$microglia to $\mathrm{CCR} 2^{+} \mathrm{CX} 3 \mathrm{CR} 1^{+}$ microglia acquiring deleterious phenotype as toxic conversion (i.e., CCR2 as a marker of neuroinflammation). Finally, the combination of $\mathrm{CCR} 2^{+}$cells (infiltrating monocytes, microglia, and neurons) might form a vicious cycle of neuroinflammation through CCL2-CCR2 signaling, thereby accelerating ALS pathology, in accordance with previous findings [1, 3, 12]. In fact, recent microglial transcriptional analyses demonstrated that the TREM2-APOE pathway induces dysregulation of 
A

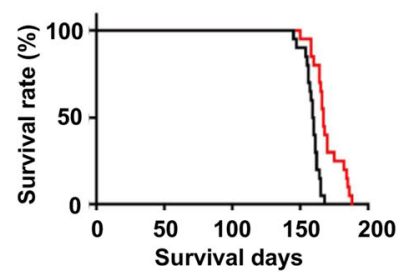

C

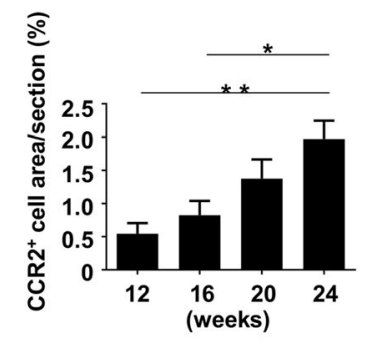

B
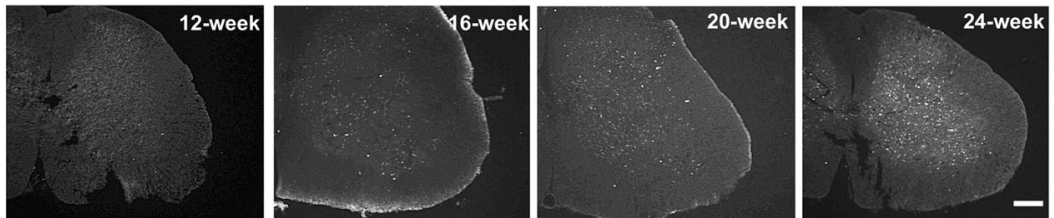

D

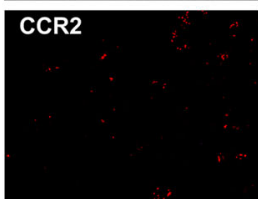

E

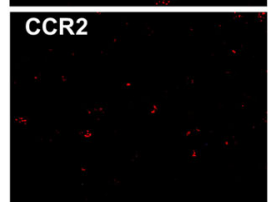

$F$
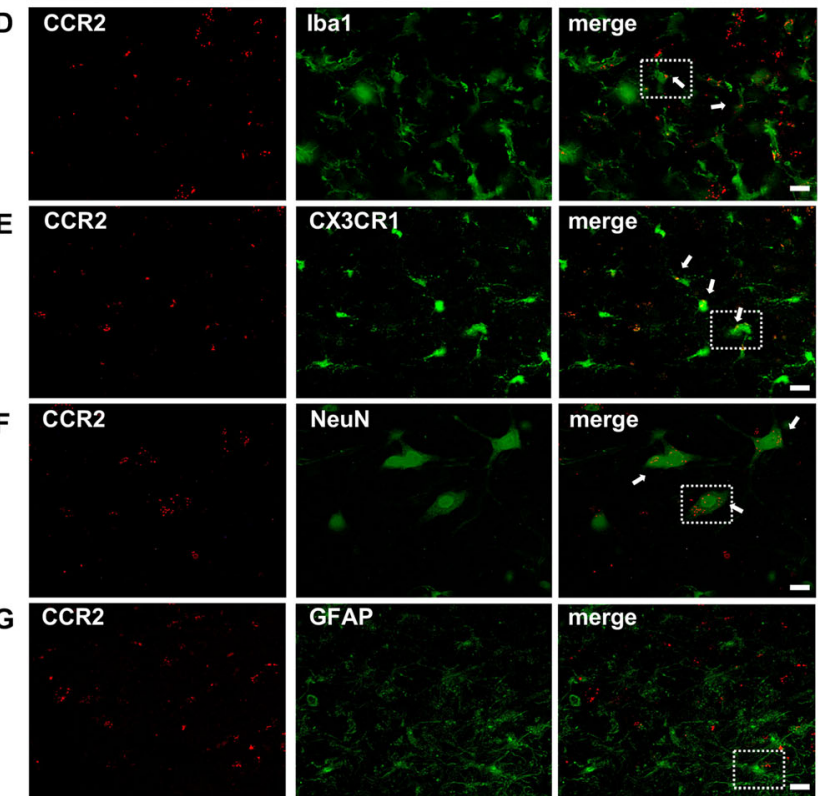

H

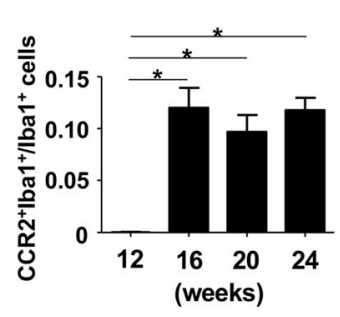

$1 \stackrel{0}{\overline{8}}$
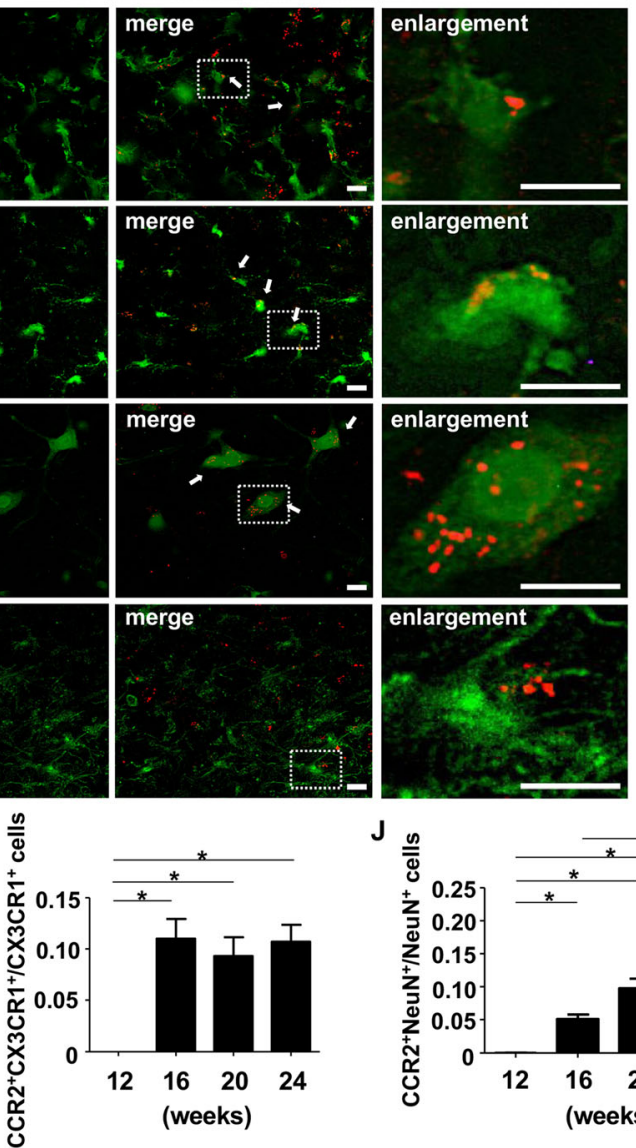

merge
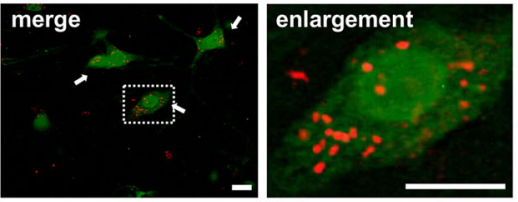

merge

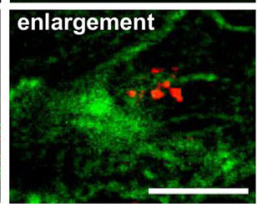

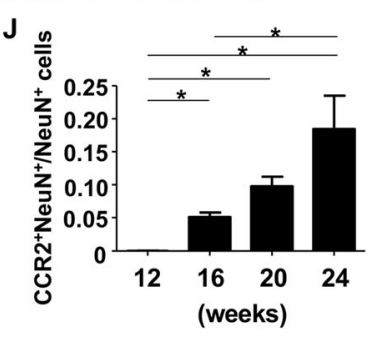

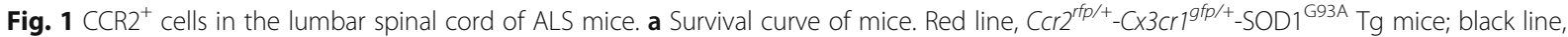

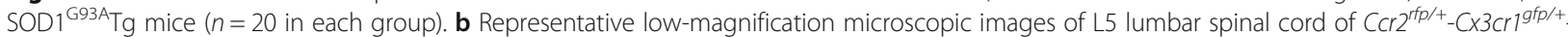
$\mathrm{SOD}_{1}{ }^{\mathrm{G} 93 \mathrm{~A}} \mathrm{Tg}$ mice. The abundance of $\mathrm{CCR}^{+}$cells increased over the course of disease progression (12-week, early stage; 16-week, middle stage; 20-week, late stage; 24-week, end stage). Scale bar, $100 \mu \mathrm{m}$. c Percentage of CCR2 ${ }^{+}$cells area in L5 lumbar spinal cord $(n=5)$. ${ }^{*}, p<0.05 .{ }^{* *}, p<$ 0.01. $\mathbf{~ d - g ~ I m m u n o f l u o r e s c e n c e ~ m i c r o g r a p h s ~ o f ~ L 5 ~ l u m b a r ~ s p i n a l ~ c o r d s ~ i n ~ 2 4 - w e e k , ~ e n d - s t a g e ~ A L S ~ m i c e ~ r e v e a l e d ~ t h a t ~ C C R 2 ~ ( r e d ) ~ w a s ~ l o c a l i z e d ~ i n ~}$ (d) infiltrating monocytes and microglia (Iba1, green), (e) microglia (CX3CR1, green), and (f) neurons (NeuN, green), but not in (g) astrocytes (GFAP, green). The right panels show enlargements of the dotted areas in the merged images in D-G. Scale bar, $10 \mu \mathrm{m}$. $\mathbf{h}-\mathbf{j}$ Percentage of CCR2 ${ }^{+}$ cells for $(\mathrm{H}) \mathrm{lba1} \mathrm{I}^{+}$infiltrating monocytes and microglia, (i) $\mathrm{CX} 3 \mathrm{CR} 1^{+}$microglia, and (j) NeuN ${ }^{+}$neurons (12-week, early stage; 16-week, middle stage: 20-week, late stage; 24-week, end stage). ${ }^{*}, p<0.05$

Cx3cr1 and homeostatic signature genes such as P2ry12, Siglech, and Tmem119 in a mouse model of neurodegenerative disease, suggesting that microglia are activated in a detrimental manner in neurodegenerative diseases [14]. Our observations also indicated that the phenotypic conversion of homeostatic $\mathrm{CX} 3 \mathrm{CR} 1^{+}$microglia to 
disease-associated $\mathrm{CCR}_{2}{ }^{+} \mathrm{CX} 3 \mathrm{CR} 1^{+}$microglia might contribute to disease progression of ALS.

The other possibility is that CNS-infiltrating $\mathrm{CCR}^{+}$ monocytes express CX3CR1. A previous study reported that chronic brain injury causes CX3CR1 upregulation in infiltrating $\mathrm{CCR}^{+}$monocytes, and that $\mathrm{CCR} 2^{+} \mathrm{CX} 3 \mathrm{CR} 1^{+}$ monocytes control their own inflammation via neuronal CX3CL1 signaling [15]. Therefore, CCR2 ${ }^{+} \mathrm{CX} 3 \mathrm{CR} 1^{+}$ monocytes may act as a self-limiting system of neuroinflammation in advanced ALS. Further studies are needed to elucidate the precise roles and mechanisms of CCR2 ${ }^{+}$ cells in ALS pathology.

In conclusion, using a $C c r 2^{r f p /+}-C x 3 c r 1^{g f p /+}$ mouse, we revealed that CCR2 expression expands from CNSinfiltrating monocytes to resident CNS cells such as microglia and neurons, but not astrocytes, over the course of ALS disease progression. This reporter mouse represents a useful and powerful tool that could provide new insights into ALS pathomechanisms.

\section{Supplementary information}

Supplementary information accompanies this paper at https://doi.org/10. 1186/s13041-020-00607-3.

Additional file 1 Activation of $\mathrm{CX}_{3} \mathrm{CR} 1^{+}$microglia in the lumbar spinal cord of ALS mice. (A) Representative low-magnification microscopic images of $\mathrm{L} 5$ lumbar spinal cord of $\mathrm{C} \mathrm{Cr} 2^{\text {rfp/t+}}-\mathrm{C} \times 3 \mathrm{Cr}^{\mathrm{gfp} /+}-\mathrm{SOD} 1^{\mathrm{G} 93 \mathrm{~A}} \mathrm{Tg}$ mice. The abundance of $\mathrm{CX} 3 \mathrm{CR}^{+}{ }^{+}$microglia increased as disease progressed (12-week, early stage; 16-week, middle stage; 20-week, late stage; 24week, end stage). Scale bar, $100 \mu \mathrm{m}$. (B) Percentage of $\mathrm{CX} 3 \mathrm{CR} 1^{+}$microglia area in L5 lumbar spinal cord $(n=5) .{ }^{*}, p<0.05$

Additional file 2 Absence of CCR2 in the lumbar spinal cord of $\mathrm{C} C r 2^{\text {rfp }} /+$ C $\times 3 c r 1^{g f p /+}$ non-Tg mice. Immunofluorescence micrographs of L5 lumbar spinal cords in 24-week $\mathrm{Cr} 2^{r f p /+}-\mathrm{C} \times 3 \mathrm{Cr} 7^{\text {gfp/+ }}$ non-Tg mice demonstrated that CCR2 (red) was not localized in (A) infiltrating monocytes and microglia (Iba1, green), (B) microglia (CX3CR1, green), (C) neurons (NeuN, green), or (D) astrocytes (GFAP, green). Scale bar, $10 \mu \mathrm{m}$.

\section{Abbreviations}

ALS: Amyotrophic lateral sclerosis; CNS: Central nervous system; SOD1: Superoxide dismutase 1

\section{Authors' contributions}

HT designed the study. HK, YH, OY, KT, AK, SK, HN, MK, MT, HD, and HT performed experiments and analyzed data. HT and TF supervised the project and experiments. HK, HT, and TF wrote the manuscript. All authors read and approved the final manuscript.

\section{Funding}

This work was supported by grants-in-aid for Scientific Research from the Ministry of Education, Culture, Sports, Science and Technology of Japan; grants from the Ministry of Health, Labour and Welfare of Japan; a grant for Strategic Research Promotion from Yokohama City University; and a grant from the Naito Foundation.

\section{Availability of data and materials}

The datasets used and/or analyzed in this study are available from the corresponding authors on reasonable request.

\section{Ethics approval and consent to participate}

This study was performed according to the Helsinki Declaration and approved by the ethical committee of Yokohama City University Graduate School of Medicine. We confirm that we have read the Journal's position on issues involved in ethical publication and affirm that this work is consistent with those guidelines.

\section{Consent for publication}

Not applicable.

\section{Competing interests}

The authors declare that they have no competing interests.

Received: 21 January 2020 Accepted: 22 April 2020

Published online: 29 April 2020

\section{References}

1. Nijssen J, Comley LH, Hedlund E. Motor neuron vulnerability and resistance in amyotrophic lateral sclerosis. Acta Neuropathol. 2017;133(6):863-85.

2. Saederup N, Cardona AE, Croft K, Mizutani M, Cotleur AC, Tsou CL, Ransohoff RM, Charo IF. Selective chemokine receptor usage by central nervous system myeloid cells in CCR2-red fluorescent protein knock-in mice. PLoS One. 2010;5(10):e13693.

3. Butovsky O, Siddiqui S, Gabriely G, Lanser AJ, Dake B, Murugaiyan G, Doykan CE, Wu PM, Gali RR, lyer LK, et al. Modulating inflammatory monocytes with a unique microRNA gene signature ameliorates murine ALS. J Clin Invest. 2012;122(9):3063-87.

4. Murdock BJ, Zhou T, Kashlan SR, Little RJ, Goutman SA, Feldman EL. Correlation of peripheral immunity with rapid amyotrophic lateral sclerosis progression. JAMA neurology. 2017:74(12):1446-54.

5. Kawaguchi-Niida M, Yamamoto T, Kato Y, Inose Y, Shibata N. MCP-1/CCR2 signaling-mediated astrocytosis is accelerated in a transgenic mouse model of SOD1-mutated familial ALS. Acta Neuropathol Commun. 2013;1:21.

6. Jung S, Aliberti J, Graemmel P, Sunshine MJ, Kreutzberg GW, Sher A, Littman DR. Analysis of fractalkine receptor CX (3) CR1 function by targeted deletion and green fluorescent protein reporter gene insertion. Mol Cell Biol. 2000; 20(11):4106-14

7. Takeuchi H, Mizoguchi H, Doi Y, Jin S, Noda M, Liang J, Li H, Zhou Y, Mori R, Yasuoka $\mathrm{S}$, et al. Blockade of gap junction hemichannel suppresses disease progression in mouse models of amyotrophic lateral sclerosis and Alzheimer's disease. PLoS One. 2011;6(6):e21108.

8. Z Zhang J, Liu Y, Liu X, Li S, Cheng C, Chen S, Le W. Dynamic changes of CX3CL1/CX3CR1 axis during microglial activation and motor neuron loss in the spinal cord of ALS mouse model. Transl Neurodegener. 2018;7:35.

9. Banisadr G, Queraud-Lesaux F, Boutterin MC, Pelaprat D, Zalc B, Rostene W, Haour F, Parsadaniantz SM. Distribution, cellular localization and functional role of CCR2 chemokine receptors in adult rat brain. J Neurochem. 2002; 81(2):257-69.

10. Gosselin RD, Varela C, Banisadr G, Mechighel P, Rostene W, Kitabgi P, MelikParsadaniantz S. Constitutive expression of CCR2 chemokine receptor and inhibition by MCP-1/CCL2 of GABA-induced currents in spinal cord neurones. J Neurochem. 2005:95(4):1023-34.

11. Jiang H, Cui H, Wang T, Shimada SG, Sun R, Tan Z, Ma C, LaMotte RH. CCL2/ CCR2 signaling elicits itch- and pain-like behavior in a murine model of allergic contact dermatitis. Brain Behav Immun. 2019;80:464-73.

12. Gyoneva S, Kim D, Katsumoto A, Kokiko-Cochran ON, Lamb BT, Ransohoff RM. Ccr2 deletion dissociates cavity size and tau pathology after mild traumatic brain injury. J Neuroinflammation. 2015;12:228.

13. Jara JH, Gautam M, Kocak N, Xie EF, Mao Q, Bigio EH, Ozdinler PH. MCP1CCR2 and neuroinflammation in the ALS motor cortex with TDP-43 pathology. J Neuroinflammation. 2019;16(1):196.

14. Krasemann S, Madore C, Cialic R, Baufeld C, Calcagno N, El Fatimy R, Beckers L, O'Loughlin E, Xu Y, Fanek Z, et al. The TREM2-APOE Pathway Drives the Transcriptional Phenotype of Dysfunctional Microglia in Neurodegenerative Diseases. Immunity. 2017:47(3):566-81 e569.

15. Morganti JM, Jopson TD, Liu S, Riparip LK, Guandique CK, Gupta N, Ferguson AR, Rosi S. CCR2 antagonism alters brain macrophage polarization and ameliorates cognitive dysfunction induced by traumatic brain injury. J Neurosci. 2015;35(2):748-60.

\section{Publisher's Note}

Springer Nature remains neutral with regard to jurisdictional claims in published maps and institutional affiliations. 